\title{
Expressed Sequence Tags and Chromosomal Localization of CDNA Clones from a Subtracted Retinal Pigment Epithelium Library
}

\author{
LINN GIESER* AND ANAND SWAROOP* ${ }^{\star} \cdot 1$ \\ *Departments of Ophthalmology and †Human Genetics, Kcllogg Eyc Center, University of Michigan, Ann Arbor, Michigan 48105
}

Received February 5, 1992; revised April 6, 1992

\begin{abstract}
Expressed sequence tags (ESTs) provide useful molecular landmarks for physical mapping and identify the position of an expressed region in the genome. The use of subtracted cDNA libraries enriched for tissue-specific genes as a source of ESTs should reduce the repetitive isolation of constitutively expressed sequences. We report here the sequence tags from the 3 '-end region of 58 new directionally cloned cDNAs from a subtracted human retinal pigment epithelium (RPE) cell line library. Eight of the cDNAs have been assigned to human chromosomes using PCR-based EST assays. Chromosomal mapping of subtracted RPE cDNA clones may also help in identifying candidate genes for inherited eye diseases. () 1992 Academic Press, Inc.
\end{abstract}

Short stretches of unique DNA sequences (sequencetagged sites, STSs) define landmarks on the genome and can be used effectively for physical mapping strategies (5). STS mapping is now recognized as a prerequisite for generating large contig maps (3). In addition to providing the same information as genomic clones, the tagged sequence sites from cDNA clones (expressed sequence tags, ESTs) also mark the position of an expressed gene. The availability of unbiased and high-fidelity directional cDNA libraries with defined orientation should maximize the output of ESTs. The sequence from the 3 -region of a cDNA frequently has no introns and shows significantly higher polymorphism between species and within different members of a family. Therefore, PCR primers from this region are useful for chromosomal localization (12), for developing polymorphic markers using single-strand conformation polymorphism (SSCP) analysis (6) or denaturing gradient gel electrophoresis (4), and for determining the expression pattern of tagged cDNA. The sequence near the poly (A) can also serve as a reference point for comparing various ESTs. Adams et al. (1) isolated 348 random ESTs from a brain library. However, this strategy is only useful in the early stages

\footnotetext{
I To whom correspondence should be addressed at 540 Kellogg Eye Center, University of Michigan, 1000 Wall Street, Ann Arbor, MI 48105.

Sequence data reported in this article have been deposited with the EMBL/GenBank Data Libraries under Accession Nos. M912161222, M91387-1403, M91480-1511, and M92283-2284.
}

of constructing the expression map of the human genome because of the repetitive isolation of highly represented and constitutively expressed genes for housekeeping functions and because of the developmental and tissue-specific expression of many genes. To reduce the redundant efforts and to obtain a complete expression map, the cDNA libraries from human tissues may be enriched for specific genes by subtraction cloning $(2,8$, 11). We have generated subtracted human retina and retinal pigment epithelium (RPE) cell line cDNA libraries by eliminating most of the constitutively expressed genes using a biotin-based procedure (11). Identification of ESTs for 58 novel cDNAs from the subtracted RPE library and chromosomal localization of 8 of these clones using polymerase chain reaction (PCR)-based EST assays are reported here.

A versatile phage vector, Charon $\mathrm{BS}$, has earlier been described for the preparation of directional cDNA libraries and their efficient transfer to Bluescript plasmid (10). Because of a simple linker-ligation strategy, most cDNA clones in Charon BS libraries contain a stretch of dAs at their $3^{\prime}$-end (generally from the poly(A) tail). Directional and size-selected cDNA libraries for more than 10 human tissues and cell lines have been generated in this vector (A. Swaroop, unpublished data). The fidelity of these libraries was evaluated by isolating cDNA clones for both known and novel genes. Using a biotinbased procedure, a human RPE cell line (0041) cDNA library was enriched for specific clones by subtraction against a mixture of RNA from fetal eye and JY lymphoblastoid cDNA libraries (11). The expression analysis of over 25 random cDNA clones from the subtracted library revealed that more than $80 \%$ of these are not detected in the JY library, and at least 4 newly identified clones are expressed only in RPE (N. Agarwal, L. Gieser, M. DelMonte, and A. Swaroop, manuscript in preparation).

To generate ESTs from the 3 '-ends, random cDNA clones from the subtracted RPE library (in Bluescript plasmid) were sequenced with T3 primer using the dideoxy chain-termination method, as described (9). The comparison of $108 \mathrm{cDNA}$ sequences to GenBank (Version 69.0) and EMRL (Version 28.0) databases using FASTA (7) showed no cDNA clone with entirely repetitive sequence, polyadenylate insert, or mitochondria- 
encoded sequence. Of these cDNAs, 36 represent 28 unique clones that are identical to human genes or are the human homologs of vertebrate genes (T'able 1). Eleven cDNAs are partially homologous to human repeat sequences or to ribosomal RNAs. Among the 61 clones with no homology to databases, only 3 were observed twice. The sequences of 58 new cDNA clones have been submitted to the GenBank Data Library.

The sequence information from eight of the new cDNAs was used to design the PCR primers using the OLIGO primer analysis software (National Biosciences, Plymouth, MN). The oligonucleotides, synthesized on a DNA synthesizer (Applied Biosystems), were used with- out purification. The PCR amplification was performed using the Gene-Amp kit and a thermocycler from Perkin-Elmer/Cetus. Annealing conditions (shown in 'Table 2) yielding a human-specific product were optimized before somatic cell hybrid analysis. The amplification products were visualized by ethidium bromide staining after agarose gel electrophoresis (NuSieve 3:1, FMC Bioproducts, Rockland, ME). DNAs from somatic cell hybrid panels ( 1 and 2) were purchased from NIGMS repository (Camden, NJ). The description of the hybrids is provided in the catalog of the repository. Each of the EST primer pairs was used to amplify a human-specific product from the DNAs of a panel of 18 human-rodent

TABLE 1

\section{Similarity of ESTs to Genes in GenBank/EMBL Databases}

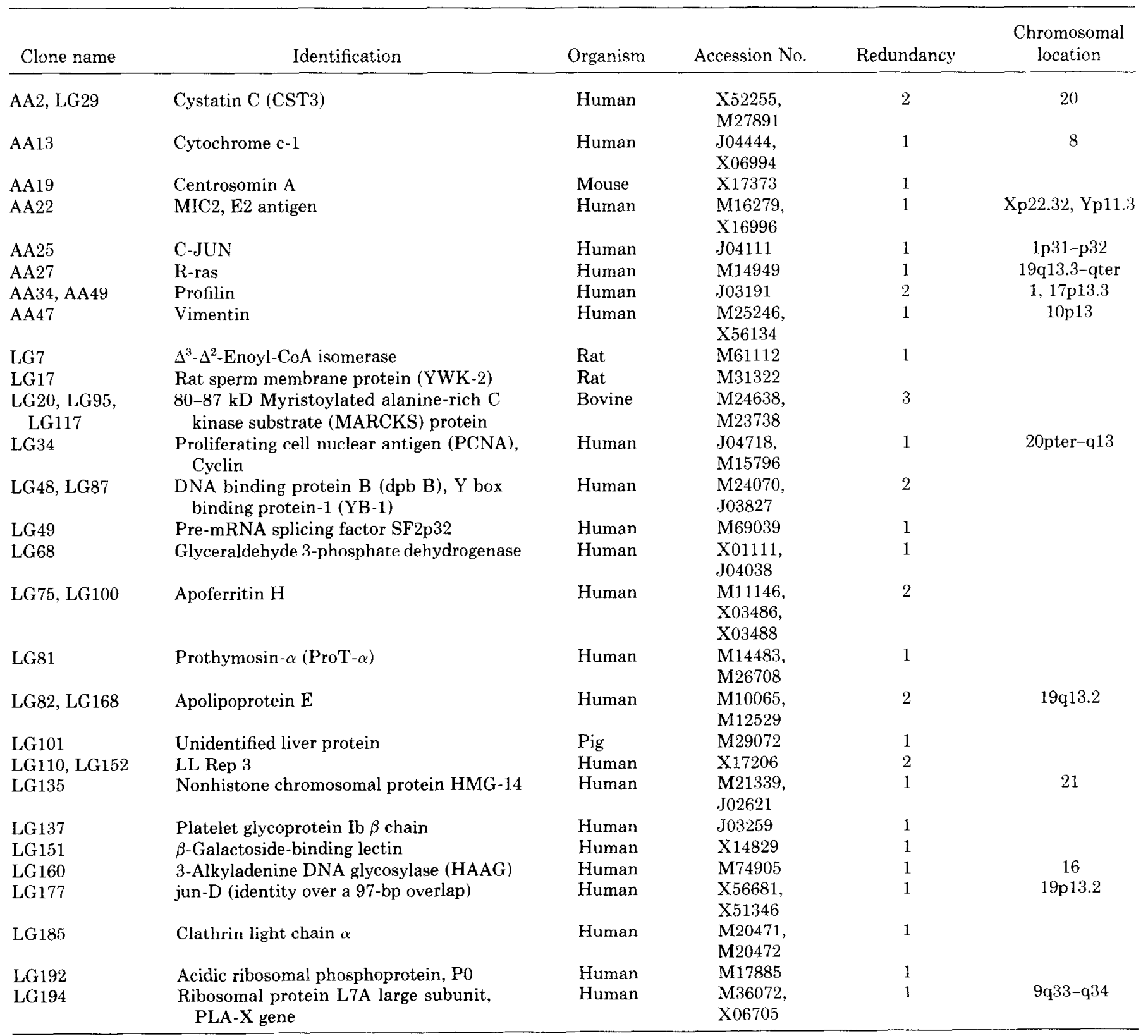




\section{TABLE 2}

PCR Primers, Conditions for Amplification, and Chromosomal Localization of Expressed Sequence Tags

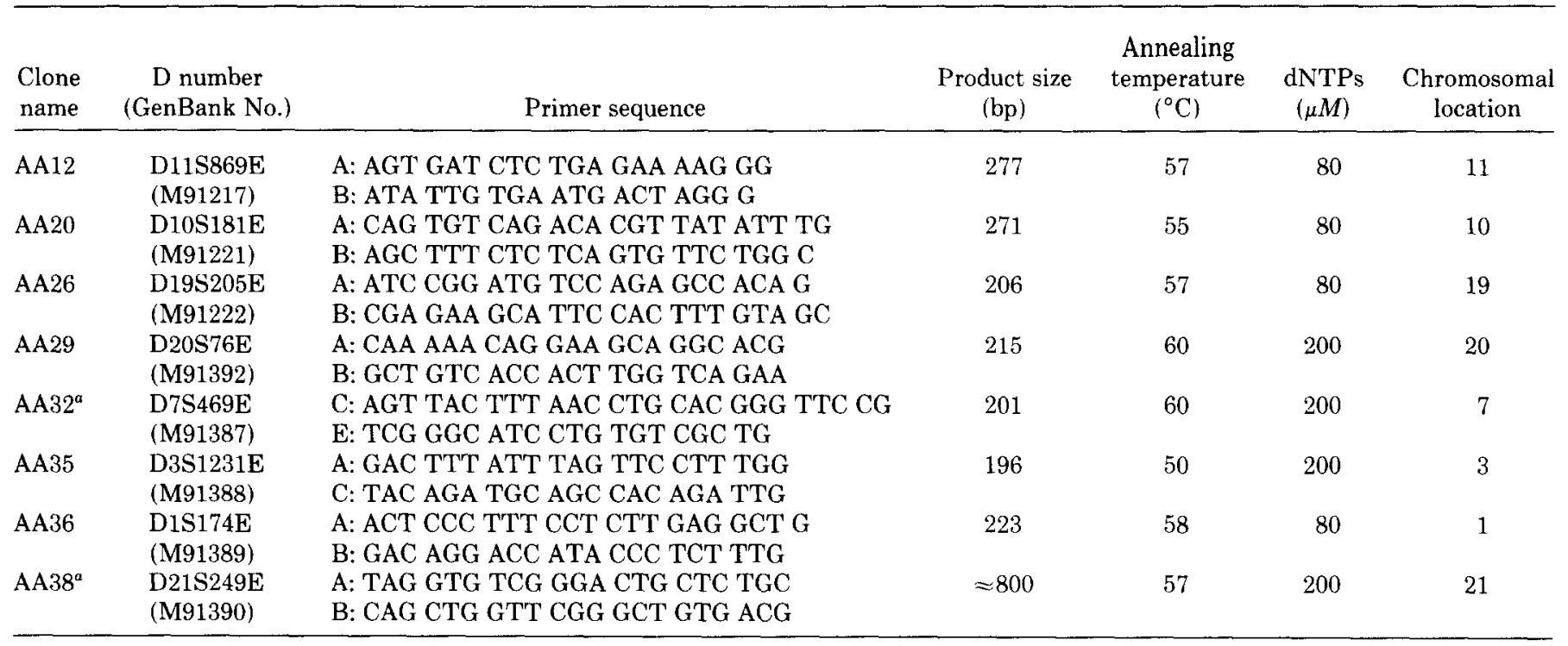

Note. $\mathrm{MgCl}_{2}$ concentration $1 \mathrm{mM}$, except $2 \mathrm{mM}$ for AA12 and $3 \mathrm{mM}$ for AA38. 50 - $\mu$ l reactions, Amplitaq $1.25 \mathrm{U} / \mathrm{rxn}$, primer concentration 0.2 $\mu M$, DNA template concentration $100 \mathrm{ng} / \mathrm{rxn}$.

$200 \mathrm{ng} / \mathrm{rxn}$ template DNA.

somatic cell hybrids (panel 1). Monochromosomic human-rodent hybrid panel 2 was used only to confirm the localization of AA38 cDNA. The presence or absence of the human-specific PCR product was then correlated with the content of human chromosomes in that particular hybrid, and an EST showing no discordancy was then assigned to a particular chromosome. The results of the chromosomal mapping studies and the D-number assignments for the eight new cDNAs are also shown in Table 2.

The major problems in developing a complete EST map of the human genome are the poor quality cDNA libraries and repetitive isolation of constitutively expressed genes. High quality cDNA libraries enriched for tissue-specific genes by subtraction cloning can minimize the redundant efforts and should provide an excellent source for generating new EST markers. The sequence tags for 58 new cDNA clones from a subtracted RPE library are reported here. The sequence analysis confirms that most of the constitutive genes have been eliminated by subtraction. Several of the known genes listed in Table 1 appear to be involved in cell growth and proliferation and are probably expressed in the RPE cell line but not in the tissue in vivo.

The chromosomal localization of ESTs using PCR assay provides valuable information for physical and genetic mapping studies. Eight of the new cDNA clones (ESTs) that are mapped in this study can now be used for sublocalization on a particular chromosome. We were unable to assign three additional ESTs to human chromosomes because of a similar size amplification product from the rodent DNA in the human-rodent somatic cell hybrids. Isolation of new ESTs from the subtracted RPE and retina libraries and their chromosomal localization should provide markers for physical mapping and may facilitate the identification of candidate genes for eye diseases.

\section{ACKNOWLEDGMENTS}

We thank Dr. Sherman M. Weissman for encouragement and Ms. Diane Sills for technical assistance during the early stages of this project. Thanks are also due to Drs. Neeraj Agarwal, Denise Yan, and Ricardo Fujita for helpful comments, Mr. Shayne Boucher for invaluable computer support, and Ms. Dorothy Giebel and Ms. Lauri Allis for the preparation of the manuscript. 'This work is supported in part hy grants from the National Institutes of Health EY-07961, the George Gund Foundation, and the Retinitis Pigmentosa Foundation, Baltimore (to A.S.).

\section{REFERENCES}

1. Adams, M. D., Kelley, J. M., Gocayne, J. D., Dubnick, M., Polymeropoulos, M. H., Xiao, H., Merril, C. R., Wu, A., Olde, B., Moreno, R. F., Kerlavage, A. R., McCombie, W. R., and Venter, J. C. (1991). Complementary DNA sequencing: Expressed sequence tags and human genome project. Science 252: 16511656.

2. Duguid, J. R., Rohwer, R. G., and Seed, B. (1988). Isolation of CDNAs of scrapie-modulated RNAs by subtractive hybridization of a cDNA library. Proc. Natl. Acad. Sci. USA 85: 5738-5742.

3. Green, E. D., Mohr, R. M., Idol, J. R., Jones, M., Buckingham, J. M., Deaven, L. L., Moyzis, R. K., and Olson, M. (1991). Systematic generation of sequence-tagged sites for physical mapping of human chromosomes: application of the mapping to human chromosome 7 using yeast artifical chromosomes. Genomics 11: 548-564.

4. Myers, R. M., Lumelsky, N., Lerman, L. S., and Maniatis, T. (1985). Detection of single base substitutions in total genomic DNA. Nature 313: 495-498. 
5. Olson, M., Hood, L., Cantor, C., and Botstein, D. (1989). A common language for physical mapping of the human genome. Science 245: 1434-1435.

6. Orita, M., Suzuki, Y., Sekiya, T., and Hayashi, K. (1989). Rapid and sensitive detection of point mutations and DNA polymorphisms using the polymerase chain reaction. Genomics 5: 874879.

7. Pearson, W. R. (1990). Rapid and sensitive sequence comparison with FASTP and FASTA. In "Methods in Enzymology" (R. F. Doolittle, Ed.), Vol. 183, pp. 63-98, Academic Press, San Diego.

8. Sive, H. L., and St. John, T. (1988). A simple subtractive hybridization technique employing photoactivatable biotin and phenol extraction. Nucleic Acids Res. 16: 10937.

9. Swaroop, A., Hogan, B. L. M., and Francke, U. (1988). Molecular analysis of the cDNA for human SPARC/Osteonectin/BM-40:
Sequence, expression, and localization of the gene to chromosome 5q31-q33. Genomics 2: 37-47.

10. Swaroop, A., and Weissman, S. M. (1988). Charon BS $(+)$ and $(-)$, versatile $\lambda$ phage vectors for constructing directional cDNA libraries and their efficient transfer to plasmids. Nucleic Acids Res. 16: 8739 .

11. Swaroop, A., Xu, J., Agarwal, N., and Weissman, S. M. (1991). A simple and efficient cDNA library subtraction procedure: isolation of human retina-specific cDNA clones. Nucleic Acids Res. 19: 1954

12. Wilcox, A. S., Khan, A. S., Hopkins, J. A., and Sikela, J. M. (1991). Use of $3^{\prime}$ untranslated sequences of human cDNAs for rapid chromosome assignment and conversion to STSs: Implications for an expression map of the genome. Nucleic Acids Res. 19: $1837-1843$. 